\title{
High-harmonic generation in spin-orbit coupled systems
}

\author{
Markus Lysne, ${ }^{1}$ Yuta Murakami, ${ }^{2}$ Michael Schüler, ${ }^{3}$ and Philipp Werner ${ }^{1}$ \\ ${ }^{1}$ Department of Physics, University of Fribourg, 1700 Fribourg, Switzerland \\ ${ }^{2}$ Department of Physics, Tokyo Institute of Technology, Meguro, Tokyo 152-8551, Japan \\ ${ }^{3}$ Stanford Institute for Materials and Energy Sciences (SIMES), SLAC National Accelerator Laboratory, Menlo Park, California 94025, USA
}

(Received 29 May 2020; revised 28 July 2020; accepted 11 August 2020; published 26 August 2020)

\begin{abstract}
We study high-harmonic generation in two-dimensional electron systems with Rashba and Dresselhaus spinorbit coupling and derive harmonic generation selection rules with the help of group theory. Based on the band structures of these minimal models and explicit simulations we reveal how the spin-orbit parameters control the cutoff energy in the high-harmonic spectrum. We also show that the magnetic field and polarization dependence of this spectrum provides information on the magnitude of the Rashba and Dresselhaus spin-orbit coupling parameters. The shape of the Fermi surface can be deduced at least qualitatively and if only one type of spin-orbit coupling is present, the coupling strength can be determined.
\end{abstract}

DOI: 10.1103/PhysRevB.102.081121

Introduction. The concept of high-harmonic generation (HHG) has attracted interest for decades in atomic systems, and lately also in the condensed matter community [1-4]. It is a nonlinear process in which a system driven by light at a certain frequency can give rise to emission at multiples of this fundamental frequency $[5,6]$. In the condensed matter context, one of the interesting aspects is that the harmonic spectrum carries information about the microscopic model, like the band structure or interaction parameters [6-9].

Several mechanisms have been proposed to explain the many facets of HHG. In some cases, the physics can be understood within a single-particle picture, but still necessitates the numerical evaluation of the interband polarization and intraband current in a coupled set of equations $[5,10,11]$. Which of these two processes dominates the emission has been debated for a long time, and a unified HHG mechanism applicable to a wide range of solids is still lacking [12]. Recently, the scope of HHG studies has been extended to strongly correlated systems [8,9,13-16], disordered systems [17-20], the effects of spin-polarized defects [21], spin or multiferroic systems [22,23], HHG in graphene and transition metal dicalchogenides [24,25], to mention a few.

The effect of spin-orbit coupling (SOC) on $\mathrm{HHG}$, to the best of our knowledge, has not been systematically explored. SOC is a relativistic effect in solids which locks the spin direction in relation to the electron momentum [26-28]. It acts on the electron's motion like an effective momentumdependent magnetic field and gives rise to an intrinsic spin Hall effect $[26,29]$. SOC plays an important role in topological insulators [30] and HHG has been used as a tool for detecting topological properties such as the Berry curvature [31]. In this Rapid Communication, we however want to study the effect of SOC in an isolated way, focusing on minimal models of two-dimensional (2D) SOC systems. The goal is to understand how SOC affects the HHG cutoffs and how the type of SOC and the coupling parameters can be extracted from characteristic features of the spectrum. For our analysis, we will adapt the existing theory for harmonic generation (HG) selection rules to models defined in momentum space [32,33], using concepts similar to nonsymmorphic symmetries in Floquet topological insulators [34]. While this type of symmetry analysis has been used before [33,35,36], it is formulated here in a way which is convenient for SOC systems.

Model and symmetries. Several previous works have discussed the Rashba and Dresselhaus Hamiltonians in a tightbinding framework [37-39], which yields

$$
\begin{aligned}
\hat{H}= & \sum_{\boldsymbol{k}} \Psi_{\boldsymbol{k}}^{\dagger}\left\{\epsilon(\boldsymbol{k}) \otimes \sigma_{0}-\left[\alpha \sin \left(k_{y} a\right)-\gamma \sin \left(k_{x} a\right)\right] \otimes \sigma_{x}\right. \\
& \left.+\left[\alpha \sin \left(k_{x} a\right)-\gamma \sin \left(k_{y} a\right)\right] \otimes \sigma_{y}+B \sigma_{z}\right\} \Psi_{k}
\end{aligned}
$$

with $\Psi_{\boldsymbol{k}}=\left(\hat{c}_{\boldsymbol{k}, \uparrow}, \hat{c}_{\boldsymbol{k}, \downarrow}\right)^{T}$ a spinor combining the annihilation operators for momentum $\boldsymbol{k}$ and spin up and down, $\epsilon(\boldsymbol{k})=$ $2 t_{h}\left[4-\cos \left(k_{x} a\right)-\cos \left(k_{y} a\right)\right]$ the dispersion of the lattice, $\sigma_{0}$ the identity matrix, and $\sigma_{i=x, y, z}$ the Pauli matrices. $\alpha$ denotes the strength of the Rashba SOC, $\gamma$ that of the Dresselhaus SOC [40], and $B$ an external magnetic field and/or exchange field $[41,42]$, which is assumed to couple only to the spin (no Landau levels). $a$ and $t_{h}$ are the lattice spacing and the hopping parameter, respectively, and we will set both to 1 in the following, i.e., energy is expressed in units of $t_{h}$. Justifications for applying the tight-binding model at high momenta can be found in, e.g., Refs. $[43,44]$, where topological invariants involving quantities defined over the entire Brillouin zone are considered. This type of SOC represents the most typical form in 2D materials [45]. We incorporate the electric field through the Peierls substitution $\boldsymbol{k} \rightarrow \boldsymbol{k}+\boldsymbol{A}(t)$, where $\boldsymbol{A}(t)$ denotes the vector potential. When developing selection rules for the HHG spectra, we will assume an AC field driving with frequency $\Omega=\frac{2 \pi}{T}$, so that the Hamiltonian satisfies $\hat{H}(t)=$ $\hat{H}(t+T)$. Following Refs. [32,33] we combine operators acting on space, time, and spin to define symmetry operations 
for the periodically driven system. This analysis is applicable to Hamiltonians of the general form $\hat{H}(t)=\sum_{\boldsymbol{k}} \Psi_{\boldsymbol{k}}^{\dagger} h(\boldsymbol{k}, t) \Psi_{\boldsymbol{k}}$.

The process of identifying the HG selection rules for a momentum-resolved quantity $O(k, t)$ contains the following two steps: (i) Identify a group, $G$, of symmetry operations $\hat{g}$, leaving $h(\boldsymbol{k}, t)$ invariant, i.e., $\hat{g} h(\boldsymbol{k}, t) \hat{g}^{-1}=h(\boldsymbol{k}, t)$. (ii) For $\hat{g} \in G$ analyze the restrictions on $n$ which follow from the condition $\hat{g} O(\boldsymbol{k}, t) e^{i n \Omega t} \hat{g}^{-1} \equiv O(\boldsymbol{k}, t) e^{i n \Omega t}$ for the generator $\hat{g}$ of $G$. The latter requirement is equivalent to saying that $O(\boldsymbol{k}, t) e^{i n \Omega t}$ belongs to the trivial representation of $G$ [32]. As the density matrix is also a time-dependent quantity which must be factored into the calculation of any observable, we assume that (ii) holds for the density matrix and the observable combined, i.e., $\hat{g} \rho(t) O(\boldsymbol{k}, t) e^{i n \Omega t} \hat{g}^{-1}=\rho(t) O(\boldsymbol{k}, t) e^{i n \Omega t}$.

To illustrate this formalism, let us derive the well-known result that inversion symmetry implies odd order harmonics. For this purpose we consider the Hamiltonian $\hat{H}(t)=$ $\sum_{k} \cos \left[k+A_{0} \cos (\Omega t)\right] \hat{c}_{k}^{\dagger} \hat{c}_{k}$, and, since we are interested in HHG, choose as operator $O$ the charge velocity $v(k, t)=$ $\frac{\partial}{\partial k} h(k, t)=-\sin \left[k+A_{0} \cos (\Omega t)\right]$, which yields the current $J(t)=\sum_{k} v(k, t)\left\langle\hat{c}_{k}^{\dagger} \hat{c}_{k}\right\rangle$. Whereas $h(k, t)=h(-k, t)$ in equilibrium $\left(A_{0}=0\right)$, in the presence of the drive, we need to extend the symmetry operation to include time as follows:

$$
\mathcal{P} \otimes \mathcal{T}_{2} \equiv\left\{\begin{array}{l}
k \rightarrow-k \\
t \rightarrow t+T / 2
\end{array} .\right.
$$

Clearly, the group generated by this operation is isomorphic to $\boldsymbol{Z}_{2}$ if we identify $t$ with $t+T$. Labeling the group element above as $\hat{g}$, we expand both sides of $\hat{g} v(k, t) e^{i n \Omega t} \hat{g}^{-1}=$ $v(k, t) e^{i n \Omega t} \quad$ to obtain $v(-k, t+T / 2) e^{i n \Omega(t+T / 2)}=$ $v(k, t) e^{i n \Omega t}$. Since $v(-k, t+T / 2)=-v(k, t), n$ is constrained by $e^{i n \pi}=-1$, which implies that $n$ is odd.

Returning to model (1) we begin by listing the generators of symmetry groups which are isomorphic to some cyclic group $\boldsymbol{Z}_{n}$. One symmetry which holds for both linearly and circularly polarized light is

$$
\mathcal{P} \otimes \mathcal{T}_{2} \otimes \mathcal{S}_{(-)}=\left\{\begin{array}{l}
\boldsymbol{k} \rightarrow-\boldsymbol{k} \\
t \rightarrow t+T / 2 \\
\left(\sigma_{x}, \sigma_{y}, \sigma_{z}\right) \rightarrow\left(-\sigma_{x},-\sigma_{y}, \sigma_{z}\right)
\end{array} .\right.
$$

For the Rashba-Dresselhaus model with $\alpha= \pm \gamma$, and for $B=$ 0 , we have the additional symmetry

$$
\mathcal{P} \otimes \mathcal{T}_{2} \otimes \mathcal{S}_{x, \mp y}=\left\{\begin{array}{l}
\boldsymbol{k} \rightarrow-\boldsymbol{k} \\
t \rightarrow t+T / 2 \\
\sigma_{x(y)} \rightarrow \mp \sigma_{y(x)}
\end{array},\right.
$$

also valid for circular and linear polarization. In the case of circularly polarized light, where we define $\boldsymbol{A}(t)=$ $\left(A_{x}(t), A_{y}(t)\right)=A_{0}(\sin (\Omega t), \cos (\Omega t))$, the following additional symmetries are found:

$$
\mathcal{R}_{90^{\circ}} \otimes \mathcal{T}_{4} \otimes \mathcal{S}_{ \pm 90^{\circ}}=\left\{\begin{array}{l}
\left(k_{x}, k_{y}\right) \rightarrow\left(k_{y},-k_{x}\right) \\
t \rightarrow t+T / 4 \\
\left(\sigma_{x}, \sigma_{y}, \sigma_{z}\right) \rightarrow\left( \pm \sigma_{y}, \mp \sigma_{x}, \sigma_{z}\right)
\end{array},\right.
$$

where the upper sign is for $\gamma=0$ and the lower sign for $\alpha=0$. Note that the $t \rightarrow t+T / 4$ transforms $\boldsymbol{A}$ as $\left(A_{x}, A_{y}\right) \rightarrow$ $\left(A_{y},-A_{x}\right)$. For the case where both $\alpha \neq 0$ and $\gamma \neq 0$, there is no symmetry involving $t \rightarrow t+T / 4$, because the Fermi surfaces only have a twofold rotational symmetry [26].
Selection rules. For linearly polarized light described by $A_{x}(t)=A_{0} \cos (\Omega t)$, let us consider the charge velocity

$$
\begin{aligned}
v_{x}(\boldsymbol{k}, t)= & \frac{\partial h(\boldsymbol{k}, t)}{\partial k_{x}}=2 \sin \left[k_{x}+A_{x}(t)\right] \sigma_{0} \\
& +\alpha \cos \left[k_{x}+A_{x}(t)\right] \sigma_{y}+\gamma \cos \left[k_{x}+A_{x}(t)\right] \sigma_{x}
\end{aligned}
$$

and the symmetry $g \equiv \mathcal{P} \otimes \mathcal{T}_{2} \otimes \mathcal{S}_{(-)}$. Since the spin transformation does not mix the $\sigma_{0}, \sigma_{x}$, and $\sigma_{y}$ matrices it is sufficient to consider $\hat{g} \cos \left[k_{x}+A_{x}(t)\right] e^{i n \Omega t} \sigma_{x, y} \hat{g}^{-1}=$ $\cos \left[-k_{x}-A_{x}(t)\right] e^{i n \Omega(t+T / 2)}\left(-\sigma_{x, y}\right) \stackrel{!}{=} \cos \left[k_{x}+A_{x}(t)\right] \times e^{i n \Omega t}$ $\sigma_{x, y}$, which leads to $e^{i n \pi}=-1$, i.e., odd order harmonics only. We proceed to demonstrate how the spin current-a quantity often studied in spintronics applications-is linked to HHG [28]. The momentum-resolved spin current operator is defined as [46]

$$
\mathcal{V}_{i j}(\boldsymbol{k}, t)=\frac{1}{2}\left[\sigma_{i} \cdot v_{j}(\boldsymbol{k}, t)+v_{j}(\boldsymbol{k}, t) \cdot \sigma_{i}\right],
$$

where $i, j=x, y, z$. We denote the spin current by $J_{i j}(t)=$ $N_{k}^{-1} \sum_{k} \operatorname{Tr}\left[\rho(t) \mathcal{V}_{i j}(\boldsymbol{k}, t)\right]$ with $N_{\boldsymbol{k}}$ the number of $\boldsymbol{k}$ points in the Brillouin zone. Using the group generator $\hat{g} \equiv \mathcal{P} \otimes \mathcal{T}_{2} \otimes$ $\mathcal{S}_{(-)}$on

$$
\mathcal{V}_{y x}(\boldsymbol{k}, t)=\gamma \cos \left[k_{x}+A_{x}(t)\right] \sigma_{0}+2 \sin \left[k_{x}+A_{x}(t)\right] \sigma_{y},
$$

the constraint following from $\hat{g} \mathcal{V}_{y x}(\boldsymbol{k}, t) e^{i n \Omega t} \hat{g}^{-1} \equiv$ $\mathcal{V}_{y x}(\boldsymbol{k}, t) e^{i n \Omega t}$ implies even order harmonics only. (The presence of a Dresselhaus SOC yields a nonzero $\mathcal{V}_{x x}$ component, which gives the same constraint on the harmonics.) This prediction is consistent with Ref. [47], which discussed a second harmonic signal in the spin current based on symmetry arguments. Furthermore, one can apply selection rules to $\mathcal{V}_{z y}$, which is the component relevant for the spin Hall effect [29]. As this expression is the anticommutator of a term with even harmonics and one with odd harmonic orders, the result is odd. Additional results and derivations regarding the selection rules can be found in the Supplemental Material (SM) [48].

Let us briefly mention the role of inversion symmetry. We have seen that despite the breaking of inversion symmetry (which has been linked to even order harmonics [26]) model (1) only produces odd order harmonics in the longitudinal charge velocity. To understand this let us consider the general Hamiltonian $\hat{H}(t)=\sum_{\boldsymbol{k}} \hat{c}_{\boldsymbol{k}, \alpha}^{\dagger} h_{\alpha, \beta}[\boldsymbol{k}+\boldsymbol{A}(t)] \hat{c}_{\boldsymbol{k}, \beta}$ with the indices denoting the relevant (orbital, spin, etc.) degrees of freedom. In the Rashba model, we clearly have that $h_{\alpha, \beta}[\boldsymbol{k}+\boldsymbol{A}(t)] \neq h_{\alpha, \beta}[-\boldsymbol{k}-\boldsymbol{A}(t)]$. However, as long as there exists a transformation $\hat{g}$ for which the action on the additional degrees of freedom yields $h_{\alpha, \beta}[\boldsymbol{k}+\boldsymbol{A}(t)]=$ $h_{g(\alpha), g(\beta)}[-\boldsymbol{k}-\boldsymbol{A}(t)]$, the HHG radiation is restricted to odd harmonics.

To test the selection rules, we simulate model (1) at inverse temperature $\beta=1 / k_{B} T=400$ (with $k_{B}=1$ ). We apply linear and circularly polarized pulses of the form

$$
\begin{aligned}
A(t)= & \frac{E_{0}}{\Omega} \sin \left[\Omega\left(t-t_{\text {avg }}\right)\right] \\
& \times \cos ^{2}\left(\frac{\Omega\left(t-t_{\text {avg }}\right)}{2 M}\right)\{\theta(t)-\theta(t-M T)\},
\end{aligned}
$$




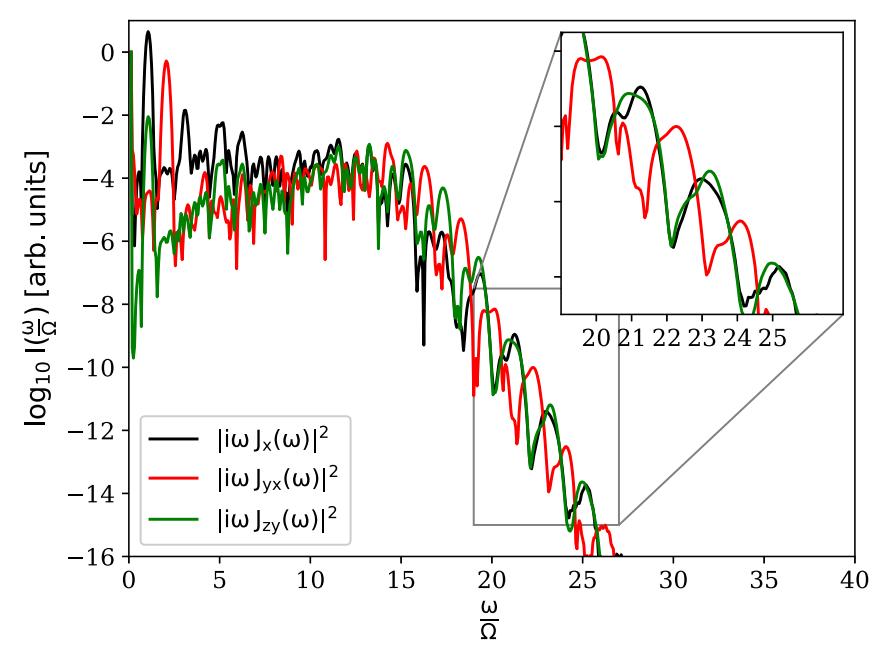

FIG. 1. High-harmonic spectra for the charge current, $J_{x}$, and two components of the spin current, $J_{y x}$ and $J_{z y}$, of the Rashba model with $\alpha=2.5, \gamma=0, \mu=3.5$, and $B=0$. A linearly polarized light field with $E_{0}=0.2$, central frequency $\Omega=0.3$, and polarization along the $x$ direction is used.

where $E_{0}$ is the electric field strength, $\Omega$ the central frequency, $M$ the number of cycles, $T$ the period, and $t_{\text {avg }}=\frac{T M}{2}$. High-harmonic spectra are calculated through the formula $\left|i \omega J_{i}(\omega)+\frac{\omega^{2}}{c} M_{i}(\omega)\right|^{2}$ where $J_{i}(\omega)$ and $M_{i}(\omega)$ denote the Fourier transform of the charge current $J_{i}(t)=N_{\boldsymbol{k}}^{-1} \sum_{\boldsymbol{k}} \operatorname{Tr}\left[\rho(t) v_{i}(\boldsymbol{k}, t)\right]$, and magnetization, $M_{i}(t)=$ $N_{k}^{-1} \sum_{k} \operatorname{Tr}\left[\rho(t) \frac{\sigma_{i}}{2}\right]$, in the $i=x, y, z$ direction, respectively. $c$ is 137 in our units. We calculate the spectra for the spin current as $\left|i \omega J_{i j}(\omega)\right|^{2}$. Prior to the Fourier transform, we apply a Blackman window to all quantities. This is given as $f_{B}(t)=$ $0.42-0.5 \cos \left(\frac{2 \pi t}{M T}\right)+0.08 \cos \left(\frac{4 \pi t}{M T}\right)$ [22]. Care must be taken with the spin current as it has a DC component [46]. Here, the DC contribution at $t=0$ is subtracted before applying the Blackman window.

Figure 1 displays the numerically obtained HHG spectra for two different components of the spin current, $J_{y x}$ and $J_{z y}$, as well as the charge current $J_{x}$. (Note that the magnetization yields no contribution to the radiation emitted along the $x$ direction when $\gamma=0$. Hence, the black line represents the total spectrum.) To the right of the plateau, we see as expected that both $J_{x}$ and $J_{z y}$ display odd order harmonics, whereas $J_{y x}$ displays even order harmonics.

$H H G$ cutoffs. We will next demonstrate how the energy scale related to SOC, $\alpha$, manifests itself in the HHG spectra. (Swapping $\alpha$ and $\gamma$ does not produce any change in the position of the cut-offs.) A linearly polarized pulse is applied along the $x$ direction and the chemical potential is set to $\mu=\epsilon(\boldsymbol{k}=\mathbf{0})$. As shown in the top panel of Fig. 2, for $\alpha>$ $t_{h}=1$, a plateau emerges, which increases with increasing $\alpha$. Upon diagonalizing Eq. (1) and setting $k_{y}=0$, we see that the maximum energy difference between the spin split bands is $\Delta E=2 \alpha$ for $B=0$ (see inset). In units of $\Omega$, this cutoff prediction is consistent with the plateaus in Fig. 2. In Fig. 3 we compare $\Delta E / \Omega$ to the measured cutoffs in different simulations.
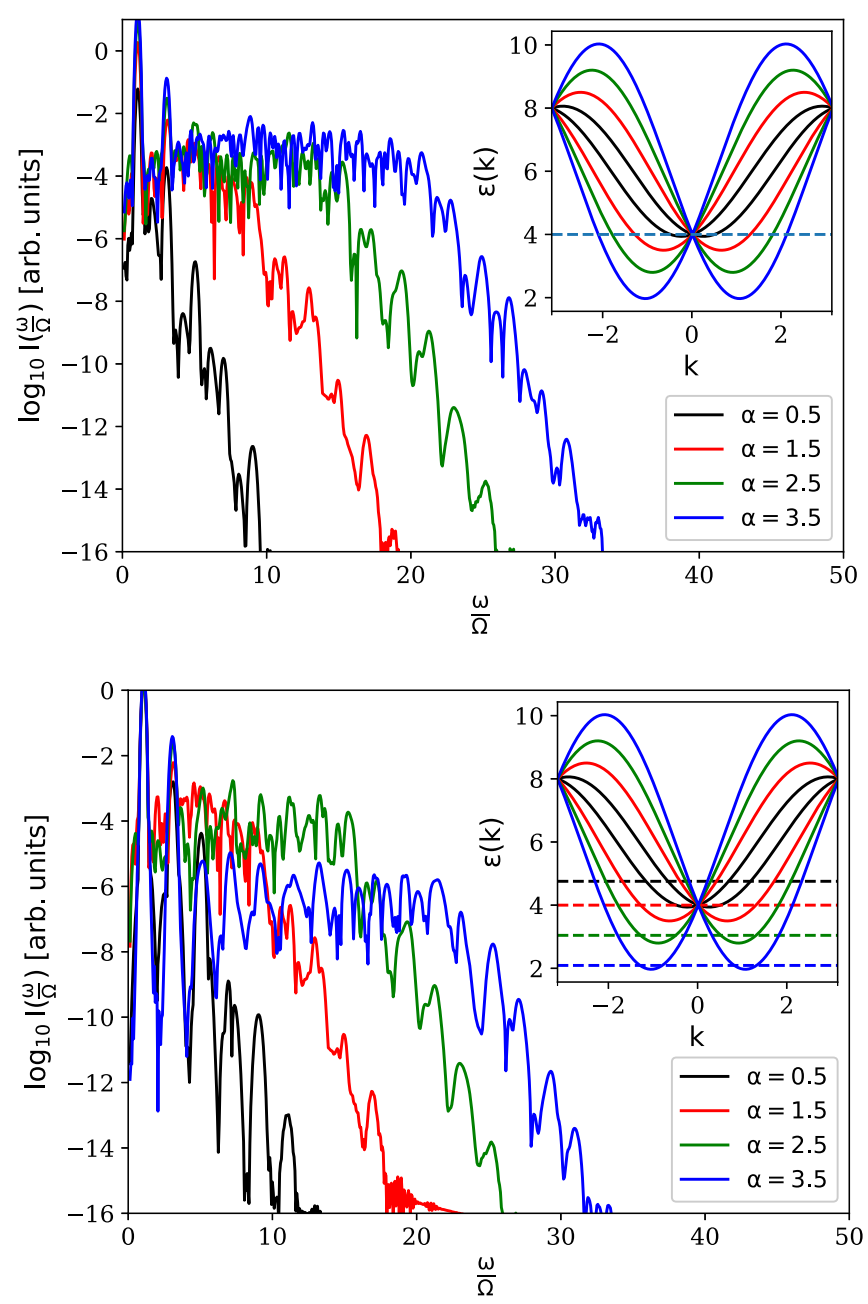

FIG. 2. $\alpha$ dependence of the HHG spectrum for $\gamma=B=0$. A linearly polarized light field with $E_{0}=0.2$, central frequency $\Omega=$ 0.3 , and polarization along the $x$ direction is used. The inset shows the two branches of the band dispersion of Eq. (1) for $k_{y}=0$. The dashed lines indicate the values of the chemical potential. In the top panel, the chemical potential is fixed at $\mu=4$, while in the bottom panel, the filling of the system is kept constant.

Whereas in the top panel of Fig. 2 the filling changes as we increase $\alpha$ while keeping $\mu$ constant, the dependence on $\alpha$ with constant filling should also be investigated.

In the lower panel of Fig. 2 we present spectra for simulations where the filling is fixed to the value corresponding to $\alpha=1.5$ and $\mu=4$. Since these cutoff scalings are very similar, one may conclude that the HHG cutoffs are controlled by the spin-orbit parameters rather than the filling.

We have also measured the cutoffs in the HHG spectra for the $J_{z y}$ component of the spin current (4), which closely follows the charge current (see also Fig. 1). The corresponding cutoff values, $f_{s c}(\alpha)$, are presented in Fig. 3 alongside those for the charge current, $f_{c c}(\alpha)$, and exhibit the same $\alpha$ dependence. Note that because there is no transverse charge current, we have a pure spin current-in line with the intrinsic spin Hall effect $[29,49]$.

Magnetic field effects. Setting $B \neq 0$ will turn model (1) into a two-band model in the basis of eigenstates of 


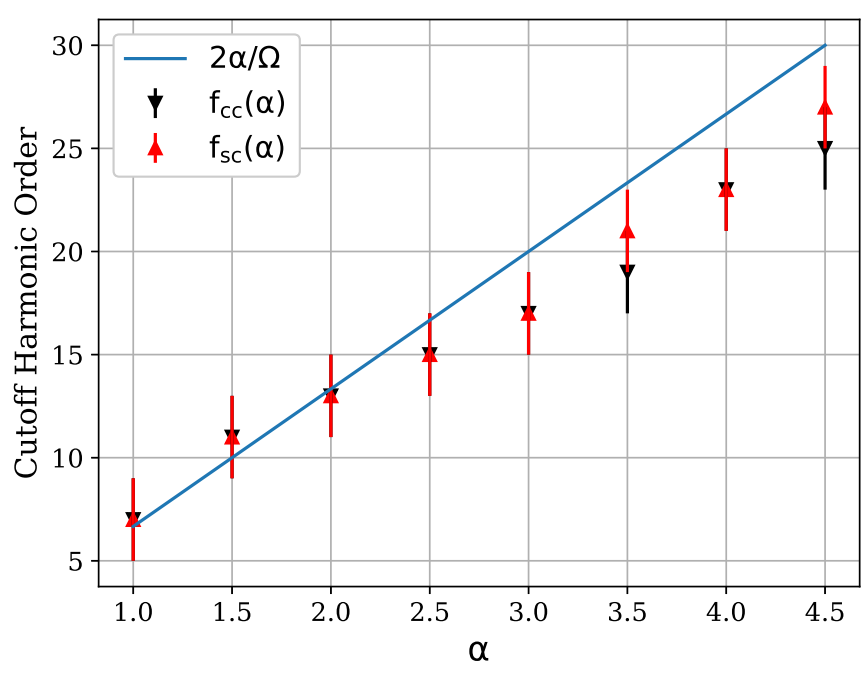

FIG. 3. HHG cutoffs for the charge current, $f_{c c}(\alpha)$, and the $J_{z y}$ spin current component, $f_{s c}(\alpha)$ when $\mu=4$ and $\gamma=B=0.2 \alpha / \Omega$ corresponds to the maximum band gap in units of $\Omega$. The uncertainty in determining the cutoff values is estimated to be $\delta f \approx 2.0$.

$\hat{S}_{z}$. Thus, for positive $B$, the lower (upper) band will be polarized in the spin-down (up) direction. Although the magnetic field/exchange field strengths considered in this section might seem high, we direct attention to a previous work where exchange fields of comparable strengths have been used to describe aspects of the anomalous Hall effect in a 2D Rashba ferromagnet [41]. SOC introduces a momentumdependent interband matrix element vanishing at the $\Gamma$ point as well as the edges of the Brillouin zone. The result is a harmonic spectrum as shown in Fig. 4. The low-order harmonics show the characteristic signature of intraband harmonics, while the grouping of harmonics starting at $\omega / \Omega>13$ can be explained by multiphoton processes across the band gap created by $B$. Indeed, the minimal band gap is $\Delta E=2 B$ so that the minimal number of photons is $2 B / \Omega \approx 13$. The

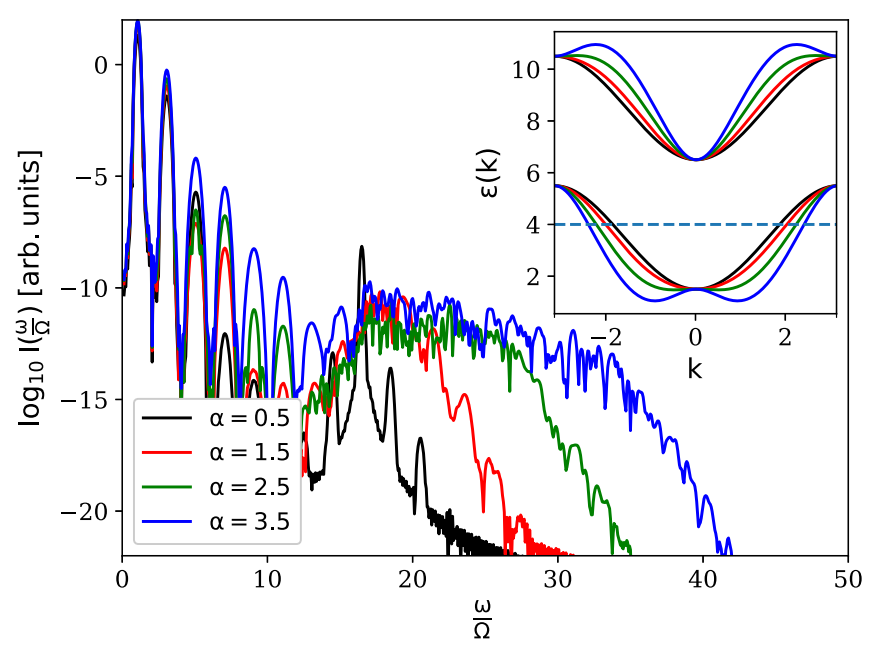

FIG. 4. $\alpha$ dependence of the high harmonic spectrum of the Rashba model with $\gamma=0.0, B=2.5$, and $\mu=4$. A linearly polarized light field with $E_{0}=0.2$, central frequency $\Omega=0.3$, and polarization along the $x$ direction is used.
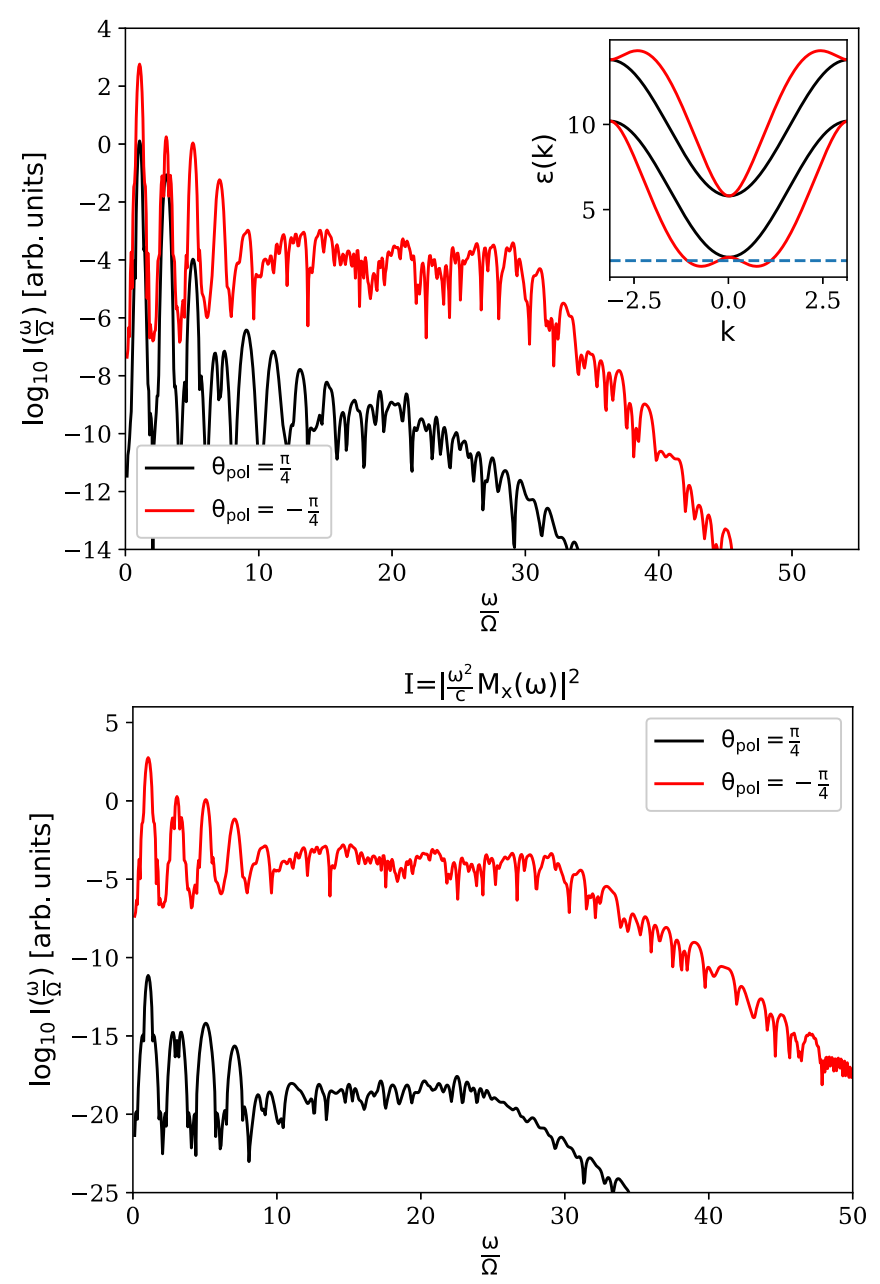

FIG. 5. Dependence of the HHG spectra on the polarization direction. The top panel shows the total spectrum, whereas the bottom panel shows the contribution from the magnetization term. The inset shows the band structure along cuts parametrized by $k\langle 1,1\rangle$ (black) and $k\langle 1,-1\rangle$ (red), i.e., along the direction of the field polarization. $\alpha=\gamma=1.5$ and $B=1.8$. The field strength is set to $E_{0}=0.5$ and $\mu=2$.

maximum band gap is $2 \sqrt{2 \alpha^{2}+B^{2}}$, which nicely explains the upper edges of the harmonic groupings in Fig. 4 (in units of $\Omega$ ). In contrast to previous HHG studies of twoband semiconductors $[10,50]$, the high-energy part of the spectrum does not exhibit a plateau structure, but rather a dome shape. We interpret this as a result of the vanishing interband coupling at the $\Gamma$ point and at the Brillouin zone boundary.

If both $\alpha$ and $\gamma$ are nonzero, the Fermi surface of model (1) has a nontrivial shape [26] and it is thus interesting to ask if the magnetic field and angular dependence of the HHG spectra allows one to extract the spin-orbit parameters. For $\alpha=\gamma$ the energy gap is bounded by $2 B \leqslant \Delta E \leqslant 2 \sqrt{B^{2}+8 \alpha^{2}}$, and we expect to see a difference in the spectra when setting the linear polarization of the fields to $\Theta_{\mathrm{pol}} \equiv \tan ^{-1}\left(A_{y} / A_{x}\right)= \pm \frac{\pi}{4}$, while measuring along the $x$ direction. The results of such calculations are shown in Fig. 5. The two spectra show the total radiation (top panel) and the magnetization contribution 
(bottom panel). A strong enhancement of harmonic intensity within the predicted plateau region is seen for $\Theta_{\text {pol }}=-\frac{\pi}{4}$ relative to $\Theta_{\text {pol }}=\frac{\pi}{4}$ in the total radiation, while the bottom panel indicates that the magnetization dynamics is the origin of this strong polarization dependence. Note also the similarity in the cutoff positions in both panels. Both for $\Theta_{\text {pol }}= \pm \frac{\pi}{4}$, the upper bound of $\Delta E$ coincides well with the observed cutoffs. To demonstrate that these results are generic for the given Hamiltonian, we present a simulation with $\mu=4$ in Fig. 4 in the SM [48].

The directional anisotropy appears because the spin expectation values (in equilibrium) are constant along lines with $\Theta_{\mathrm{pol}}=\frac{\pi}{4}$ while they vary along $\Theta_{\mathrm{pol}}=-\frac{\pi}{4}$ when $\alpha=\gamma$ [51]. The figure furthermore demonstrates that the magnetic radiation contribution is non-negligible in some cases. This also holds for the Dresselhaus model when the radiation is measured along the driving direction, as suggested by the spin-momentum locking picture. It should also be pointed out that the larger field strength used here (relative to the previous figures) implies that the electrons sample a larger region of the Brillouin zone.

Conclusions. We have explored ways of extracting SOC parameters from HHG spectra. If only a Rashba or Dresselhaus coupling is present, the coupling strength can be directly deduced from the cutoff of the HHG plateau or a characteristic grouping of harmonics in strong magnetic/exchange fields. If both couplings are nonzero, insight into the relative size of the SOC parameters can be gained by studying the polarization dependence. In particular, a large change in the HHG intensity upon rotation by $90^{\circ}$ indicates that $\alpha$ and $\gamma$ are of comparable magnitude. The general symmetry analysis for linearly and circularly polarized fields can help to determine relevant aspects of a microscopic model on the basis of HHG spectra, at least for systems with strong SOC. We have also shown that the $J_{z y}$ spin current is strongly correlated with the $J_{x}$ charge current and that both follow the same cutoff scaling with increasing $\alpha$. Since there is much interest in the control of spin currents, high-harmonic generation and detection methods may be useful for identifying SOC materials with ideal properties for spintronics applications. Further effects, such as spin relaxation through the D'yakonov-Perel' mechanism, or the influence of electron-electron scattering may warrant more detailed investigations. How interactioninduced dephasing affects the coherence needed to form a Floquet state has recently been studied in Ref. [52], and similar techniques could also be applied to models with SOC.

Acknowledgments. M.L. and P.W. acknowledge support from ERC Consolidator Grant No. 724103. Y.M. acknowledges support from Grant-in-Aid for Scientific Research from JSPS; KAKENHI Grants No. JP19K23425, No. JP20K14412, and No. JP20H05265; and JST CREST Grant No. JPMJCR1901. M.S. acknowledges financial support from the U.S. Department of Energy (DOE), Office of Basic Energy Sciences, Division of Materials Sciences and Engineering, under Contract No. DE-AC02-76SF00515, and the Alexander von Humboldt Foundation for its support with a Feodor Lynen scholarship.
[1] M. Lewenstein, P. Balcou, M. Y. Ivanov, A. L'Huillier, and P. B. Corkum, Phys. Rev. A 49, 2117 (1994).

[2] S. Ghimire and D. A. Reis, Nat. Phys. 15, 10 (2019).

[3] F. Krausz and M. Ivanov, Rev. Mod. Phys. 81, 163 (2009).

[4] S. Ghimire, A. D. DiChiara, E. Sistrunk, P. Agostini, L. F. DiMauro, and D. A. Reis, Nat. Phys. 7, 138 (2011).

[5] G. Vampa, C. R. McDonald, G. Orlando, D. D. Klug, P. B. Corkum, and T. Brabec, Phys. Rev. Lett. 113, 073901 (2014).

[6] T. T. Luu, M. Garg, S. Y. Kruchinin, A. Moulet, M. T. Hassan, and E. Goulielmakis, Nature (London) 521, 498 (2015).

[7] G. Vampa, T. J. Hammond, N. Thiré, B. E. Schmidt, F. Légaré, C. R. McDonald, T. Brabec, D. D. Klug, and P. B. Corkum, Phys. Rev. Lett. 115, 193603 (2015).

[8] Y. Murakami, M. Eckstein, and P. Werner, Phys. Rev. Lett. 121, 057405 (2018).

[9] M. Lysne, Y. Murakami, and P. Werner, Phys. Rev. B 101, 195139 (2020).

[10] D. Golde, T. Meier, and S. W. Koch, Phys. Rev. B 77, 075330 (2008).

[11] M. Hohenleutner, F. Langer, O. Schubert, M. Knorr, U. Huttner, S. Koch, M. Kira, and R. Huber, Nature (London) 523, 572 (2015).

[12] H. Liu, Y. Li, Y. S. You, S. Ghimire, T. F. Heinz, and D. A. Reis, Nat. Phys. 13, 262 (2017).

[13] R. Silva, I. V. Blinov, A. N. Rubtsov, O. Smirnova, and M. Ivanov, Nat. Photonics 12, 266 (2018).

[14] Y. Murakami and P. Werner, Phys. Rev. B 98, 075102 (2018).
[15] S. Imai, A. Ono, and S. Ishihara, Phys. Rev. Lett. 124, 157404 (2020).

[16] N. Tancogne-Dejean, M. A. Sentef, and A. Rubio, Phys. Rev. Lett. 121, 097402 (2018).

[17] C. Yu, K. K. Hansen, and L. B. Madsen, Phys. Rev. A 99, 063408 (2019).

[18] S. Almalki, A. M. Parks, G. Bart, P. B. Corkum, T. Brabec, and C. R. McDonald, Phys. Rev. B 98, 144307 (2018).

[19] G. Orlando, C.-M. Wang, T.-S. Ho, and S.-I. Chu, J. Opt. Soc. Am. B 35, 680 (2018).

[20] K. Chinzei and T. N. Ikeda, Phys. Rev. Res. 2, 013033 (2020).

[21] M. Mrudul, N. Tancogne-Dejean, A. Rubio, and G. Dixit, npj Comput. Mater. 6, 10 (2020).

[22] S. Takayoshi, Y. Murakami, and P. Werner, Phys. Rev. B 99, 184303 (2019).

[23] T. N. Ikeda and M. Sato, Phys. Rev. B 100, 214424 (2019).

[24] N. Yoshikawa, T. Tamaya, and K. Tanaka, Science 356, 736 (2017).

[25] N. Yoshikawa, K. Nagai, K. Uchida, Y. Takaguchi, S. Sasaki, Y. Miyata, and K. Tanaka, Nat. Commun. 10, 3709 (2019).

[26] A. Manchon, H. C. Koo, J. Nitta, S. Frolov, and R. Duine, Nat. Mater. 14, 871 (2015).

[27] A. Hirohata, K. Yamada, Y. Nakatani, L. Prejbeanu, B. Diény, P. Pirro, and B. Hillebrands, J. Magn. Magn. Mater. 509, 166711 (2020)

[28] I. Žutić, J. Fabian, and S. Das Sarma, Rev. Mod. Phys. 76, 323 (2004). 
[29] S.-Q. Shen, Phys. Rev. B 70, 081311(R) (2004).

[30] B. A. Bernevig and T. L. Hughes, Topological Insulators and Topological Superconductors (Princeton University Press, Princeton, NJ, 2013).

[31] T. T. Luu and H. J. Wörner, Nat. Commun. 9, 916 (2018).

[32] O. E. Alon, V. Averbukh, and N. Moiseyev, Phys. Rev. Lett. 80, 3743 (1998).

[33] O. Neufeld, D. Podolsky, and O. Cohen, Nat. Commun. 10, 405 (2019).

[34] T. Morimoto, H. C. Po, and A. Vishwanath, Phys. Rev. B 95, 195155 (2017).

[35] N. Saito, P. Xia, F. Lu, T. Kanai, J. Itatani, and N. Ishii, Optica 4, 1333 (2017).

[36] T. N. Ikeda, K. Chinzei, and H. Tsunetsugu, Phys. Rev. A 98, 063426 (2018).

[37] F. Mireles and G. Kirczenow, Phys. Rev. B 64, 024426 (2001).

[38] T. Pareek and P. Bruno, Pramana 58, 293 (2002).

[39] T. P. Pareek and P. Bruno, Phys. Rev. B 63, 165424 (2001).

[40] E. I. Rashba, Phys. E (Amsterdam, Neth.) 34, 31 (2006).

[41] I. A. Ado, I. A. Dmitriev, P. M. Ostrovsky, and M. Titov, Phys. Rev. Lett. 117, 046601 (2016).
[42] A. Qaiumzadeh, R. A. Duine, and M. Titov, Phys. Rev. B 92, 014402 (2015).

[43] S.-Q. Shen, Topological Insulators (Springer, New York, 2012), Vol. 174.

[44] C. L. Kane and E. J. Mele, Phys. Rev. Lett. 95, 146802 (2005).

[45] R. Winkler, S. Papadakis, E. De Poortere, and M. Shayegan, Spin-Orbit Coupling in Two-Dimensional Electron and Hole Systems (Springer, New York, 2003), Vol. 41.

[46] E. I. Rashba, Phys. Rev. B 68, 241315(R) (2003).

[47] K. Hamamoto, M. Ezawa, K. W. Kim, T. Morimoto, and N. Nagaosa, Phys. Rev. B 95, 224430 (2017).

[48] See Supplemental Material at http://link.aps.org/supplemental/ 10.1103/PhysRevB.102.081121 for the derivation of certain selection rules and additional simulation data.

[49] J. Sinova, D. Culcer, Q. Niu, N. A. Sinitsyn, T. Jungwirth, and A. H. MacDonald, Phys. Rev. Lett. 92, 126603 (2004).

[50] M. Wu, S. Ghimire, D. A. Reis, K. J. Schafer, and M. B. Gaarde, Phys. Rev. A 91, 043839 (2015).

[51] M.-H. Liu, K.-W. Chen, S.-H. Chen, and C.-R. Chang, Phys. Rev. B 74, 235322 (2006).

[52] M. Schüler, U. De Giovannini, H. Hübener, A. Rubio, M. A. Sentef, T. P. Devereaux, and P. Werner, arXiv:2003.11621. 